\title{
Migración y fuerza de trabajo en los asentamientos humanos irregulares de la ciudad de Mexicali, B. C. 1940-1982
}

\author{
David F. Fuentes Romero \\ Instituto de Investigaciones Sociales \\ Universidad Autónoma de Baja California
}

\section{Resumen}

La región fronteriza del Norte en general es reflejo del tipo de relaciones entre México y Estados Unidos. En este documento se presenta información que permite constatar la importancia de los asentamientos humanos irregulares en el proceso de urbanización de la frontera, y en el conjunto de desplazamientos de población entre México y Estados Unidos. Además se detectó que dichos asentamientos juegan un papel destacado en el proceso migratorio en dos planos convergentes: a nivel internacional, entre México y los Estados Unidos; y a nivel regional, entre los municipios de Baja California y los del noroeste de Sonora. La investigación de este fenómeno migratorio aportó datos relevantes y sugirió cuestiones de gran importancia cuya respuesta es necesaria y por ende plantea la pertinencia de seguir avanzando en el estudio del tema.

Palabras clave: migración, asentamientos humanos irregulares, crisis agrícola, Programa Nacional Fronterizo (PNF), Programa Industrial Fronterizo (PIF).

\begin{abstract}
The Northern border region generally reflects the kind of relations between Mexico and the United States. This document presents information that allows us to prove the importance of irregular human settlements during the urbanization process of the border, and in the group of population displacements between Mexico and the United States. Besides that, it was discovered that said settlements played a prominent role in the migratory process at two converging levels: the international one, between Mexico and the United States; and the regional one, between the municipalities of Baja California and the Northeast of Sonora. The study of this migratory phenomenon provided critical information and suggested important issues that demand the proper answer while requesting to continue researching on this topic.
\end{abstract}

Keywords: migration, irregular human settlements, crisis of agriculture, Border National Program (PNF), Border Industrial Program (PIF). 


\title{
MIGRACION Y FUERZA DE TRABAJO EN LOS ASENTAMIENTOS HUMANOS IRREGULARES DE LA CIUDAD DE MEXICALI*

\author{
B. C. 1940 - 1982.
}

\author{
Por \\ David F. Fuentes Romero \\ Investigador del Instituto de Investigaciones \\ Sociales UABC
}

\section{INTRODUCCION.}

El estudio de los asentamientos humanos irregulares desde la perspectiva de análisis que considera a los movimientos migratorios internos como un fenómeno de carácter histórico-estructural, implica por una parte, desplazar el énfasis de la indagación de los aspectos subjetivos que racionalizan estos procesos migratorios, sin necesidad de excluirlos totalmente, hacia la identificación de los rasgos sociales de los grupos que participan en tales movilizaciones. Por otra parte, esta forma de aproximación permite abordar la temática referida a los efectos económicos y sociales que generan estas corrientes migratorias internas, en los lugares de destino de las mismas.

Respecto a estas corrientes migratorias internas en México, se han señalado dos rasgos característicos; el primero concierne a su orientación hacia un reducido número de localidades urbanas del país, las cuales a pesar de incrementarse en términos absolutos, disminuyen en términos relativos al total de localidades de la nación. 1

El segundo rasgo de estos movimientos poblacionales, se refiere, básicamente, a los efectos que tal concentración de la migración interna ha generado en este reducido número de localidades. Es decir, señala el hecho de que "... la concentración de los migrantes en unos pocos centros urbanos significó la disponibilidad de una fuerza de trabajo abundante... pero también ha propiciado la constitución de crecientes masas marginales en los grandes núcleos urbanos". 2

En el conjunto de localidades que se caracterizan para atraer las corrientes de migracion interna, las ciudades de la frontera norte de México han juzado un papel importante.

Una de las consecuencias más evidentes, derivada de este proceso inmizratorio, ha sido el surgimiento y consolidación de una gran cantidad de

\footnotetext{
- Este artículo forma parte del proyecto de investigación "Asentamientos Humanos Irregulares en Baja California", del Instituto de Investigaciones Sociales de la Universidad Autónoma de Baja California.
}

'Unikel, Luis. El Desarrollo Urbano de México. El Colegio de México CEED. 2dz. Edición, 1978. México, D.F. pp. 48-49.

'Alba. Francisco. La Población de México: Evolución y Dilema. El Colegio de México, CEED. 2da. Edición, 1979. México, D.F. pp. 162-163. 
asentamientos humanos irregulares reconocidos oficialmente. Ahora bien, a pesar de la magnitud e importancia que ha adquirido este fenómeno en la ciudad de Mexicali, se observa que a éste no se le ha dedicado la atención pertinente. Más aún, en términos de investigación sobre asentamientos humanos irregulares en torno al desarrollo urbano que se presenta en la frontera, apenas comienza a cobrar interés en el ámbito de la investigación social. No obstante esta limitante, el examen del asentamiento humano fronterizo, particularmente en lo que toca al proceso de urbanización, tiene importancia debido al papel preponderante que tiene ante la creciente concentración de población y a la actividad económica específica desarrollada en las áreas urbanas.

En abril de 1981 el Instituto de Investigaciones Sociales de Ia Universidad Autónoma de Baja California inició un proyecto de investigación sobre los asentamientos humanos irregulares (AHI) en el área urbana de Mexicali, Baja California. El objetivo principal de este proyecto fue, por una parte, localizar a los asentamientos humanos irregulares en el área urbana de Mexicali; y por otra parte, caracterizar el fenómeno, identificando cuatro aspectos principales: estructura demográfica, ocupación e ingreso, vivienda y patrón migratorio.

La investigación de este fenómeno aportó datos relevantes y sugirió cuestiones de gran importancia cuya respuesta es necesaria y por ende plantea la pertinencia de avanzar en el estudio del tema. En particular, se detectó que los asentamientos humanos irregulares juegan un papel destacado en el proceso migratorio que ocurre en dos planos convergentes: a nivel internacional, entre México y los Estados Unidos; y a nivel regional, entre municipios de Baja California y noroeste de Sonora. En efecto, cerca del 40 por ciento de la población residente en los asentamientos humanos irregulares es población migrante, procedente del interior de la república. Una proporción elevada del 60 por ciento restante es población joven, hijos de los migrantes nacidos en Baja California. Los datos anteriores permitieron constatar la importancia de este tipo de asentamientos en el proceso de urbanización de la frontera, y en el conjunto de desplazamiento de población entre México y Estados Unidos.

En el caso de Mexicali, sin embargo, este tipo de asentamientos es más la excepción que la regla en el proceso de urbanización. El total de residentes en las 26 colonias irregulares, de acuerdo con el levantamiento realizado en 1982 , es de 21,600 habitantes que representaban cerca del 5 por ciento de la población total de la ciudad de Mexicali en ese año.

Se trata, en el caso de esta ciudad, de asentamientos permanentes con un ritmo de crecimiento relativamente alto. La población en edad de trabajar se ocupa, en la mayoría de los casos, en empleos más bien inestables, que demandan poca o nula preparación y ofrecen salarios bajos, con frecuencia inferiores al salario mínimo ( 64 por ciento de la población ocupada se encuentra en esta situación, de acuerdo con la encuesta realizada). 
Partiendo de lo anterior planteamos las siguientes hipótesis de trabajo en la investigación.

1 La movilidad interna genera una tendencia a la concentración geográfica.

2 La frontera favorece al desarrollo de los asentamientos humanos irregulares:

a) Como espacio original dentro del proceso global de movilidad interna.

b) Como punto de refugio-pasaje hacia los Estados Unidos.

El presente artículo es un resumen de los resultados obtenidos en el programa de investigación.

\section{CARACTERISTICAS ESPECIFICAS DE LA REGION FRONTERIZA EN BAJA CALIFORNIA.}

Las particularidades de la región fronteriza en Baja California están dadas por el tipo de actividades con las que se inicia la vida económica en la entidad, así como las causas que orillaron a los primeros pobladores de la región a instalarse en estas tierras.

De 1914 a 1918 a consecuencia de la I Guerra Mundial, el algodón tiene una gran demanda internacional; de 1928 a 1929 la producción algodonera fue de 80,000 pacas cultivadas en 64 mil hectáreas; debido a la crisis en 1929 hubo un descenso en la producción de algodón, provocando que para 1933 sólo se recogieran 14,000 pacas; entre 1942 y 1945 la producción algodonera superó las cifras anteriores llegando a 91,000 pacas anuales cultivadas en casi 90,000 hectáreas del valle de Mexicali. En 1946 el auge algodonero se ve afectado a causa de la caída de los precios internacionales.

En el período de 1949-1955 se reinicia de nuevo el auge algodonero debido a que, por un lado, la guerra de Corea propició el alza de los precios internacionales; y por otro, las devaluaciones del peso mexicano con relación al dólar en 1949 y 1953 provocaron que el algodón mexicano fuera más competitivo en el mercado internacional. Entre 1949 y 1955, el valle de Mexicali produjo 294 mil pacas por año "... para el ciclo de 1954-1955, Mexicali obtuvo la distinción de ser la región con mayor producción de algodón en el país..." 3

La amplia disponibilidad de agua de que gozaba el valle de Mexicali se debe al acuerdo que hubo entre Estados Unidos y México en el año de 1944, en el cual se estipulaba que Estados Unidos entregaría aguas del río Colorado para el riego de sembradíos de México, factor que fue de gran importancia para el auge algodonero.

ILadman. Jerry R. El Desarrollo de la Economia Regional de Mexicali. p. 110. 
En 1940 México adoptó un modelo de acumulación basado en la sustitución de importaciones. En base a este modelo el sector agrícola debía cumplir con ciertos requisitos indispensables, y eran: a) abastecer al mercado interno con productos de consumo terminal, b) generar la oferta requerida de materias primas para la industria de transformación, y c) captar a través de la exportación las divisas necesarias para la importación de medios de producción. El valle de Mexicali contribuyó al éxito del último requisito; es indudable que el auge algodonero propició la acumulación de capital. Parte de los excedentes obtenidos de la producción algodonera se utilizaron para promover la capitalización del sector agrícola a través de la actividad agroindustrial, tanto en la producción de insumos como en la transformación de productos, la mecanización y la incorporación de nueva tecnología.

La inmigración de fuerza de trabajo del interior del país al valle de Mexicali también ayudó al gran auge del algodón.

En 1960-1970 se da una crisis muy fuerte en el sector agrícola; por consecuencia, la producción de algodón comienza a descender aceleradamente debido a varios factores:

1. "... Se llega al conocimiento de que en el estado de Arizona, E.U.A., en la cuenca del río Gila, se había construido el distrito de drenaje 'Wellton Mohawk' con la finalidad de agotar enormes depósitos subterráneos de aguas fósiles salobres, para sustituirlas con infiltración de aguas dulces del río Colorado, en vista de reservarlas para el futuro. Los bombeos de aguas fósiles del Wellton Mohawk se estaban descargando en el cauce del río Gila tributario del Colorado, y propiciaron la contaminación de las aguas vírgenes del Colorado correspondientes a México desde el Tratado de 1944. Este hecho amenazaba inutilizar en breve plazo las tierras del valle de Mexicali. . ." 4

La inutilización de tierras por la salinidad que poco a poco iba en aumento en las aguas del río Colorado se convirtió en una amenaza para el cultivo del algodón. Los agricultores del valle presionaron al gobierno federal mexicano para que tomara medidas de solución a este problema, y sólo hasta 1973, a través de la Minuta 242 de la Comisión Internacional de Límites y Aguas se acordó que Estados Unidos enviara a México aguas que ellos mismos utilizaban y tenían guardadas en la presa de Imperial, la solución llegó muy tarde ya que las tierras del valle de Mexicali fueron afectadas de una manera total y sin remedio.

2. En 1966 se viene la plaga del gusano rosado al valle de Mexicali, el cual "... ha sido el castigo de los agricultores de algodón en muchos lugares. . los agricultores de algodón en Mexicali hicieron esfuerzos para prevenirlo colaborando con especialistas del Departamento de Agricultura de los Estados Unidos, a principios de la década de 1960, pero una vez que la plaga

4Diełach, Nicole, El Proceso de Producción Agrícola en el Valle de Mexicali.Tesis de licenciatura. Escuela de Ciencias Sociales y Politicas de la UABC.1977, pp. 111-112. 
llegó a Yuma, también estaba en Mexicali. Entró en la región en 1966 y para 1967 se había generalizado..." 5 , se utilizaron insecticidas, pero el cultivo del algodón fue cada vez de menor calidad.

3. La baja de los precios internacionales del algodón fue también uno de los factores importantes para la caída del algodón ya que en esa época entraron al mercado las fibras sintéticas sustituyendo a las fibras naturales; los precios de los derivados del petróleo permitieron la producción textil a base de fibras sintéticas siendo éstas mejor pagadas que las naturales.

En resumen, los problemas de la salinidad del agua, de la plaga del gusano rosado, de la baja de precios del algodón en el mercado internacional y las limitaciones de volúmenes de agua provocaron que la producción algodonera en el valle de Mexicali entrara en plena crisis.

La caída del algodón y el constante crecimiento de la población debido a la inmigración proveniente del centro del país, provocó una concentración de la población en las zonas urbanas dando como resultado que el $85 \%$ de la población bajacaliforniana viva en localidades de más de 2,500 habitantes. 6 El principal factor que ha acelerado el exagerado crecimiento poblacional de las ciudades fronterizas de Baja California, principalmente Tijuana y Mexicali, es la gran cantidad de gente que se asienta en estas ciudades con el fin de cruzar la frontera en busca de trabajo, aunque las actuales políticas de migración por parte del gobierno de los Estados Unidos tienden a impedir u obstaculizar el logro de tales fines, incluso hasta cierto punto "tratan de acabar" con los ilegales que trabajan en ese país, y por tal motivo, constantemente son repatriados mexicanos que trabajan al otro lado de la frontera, en calidad de ilegales, quienes se quedan a vivir en estas ciudades.

\section{POLITICAS DEL ESTADO MEXICANO ANTE EL PROBLEMA FRONTERIZO.}

Como respuesta a la grave situación de desempleo en las zonas fronterizas, en el año de 1961 el gobierno federal estableció el Programa Nacional Fronterizo (PRONAF) cuyos objetivos estaban encaminados hacia la integración de esta zona con el resto del país en lo referente a aspectos sociales y económicos, mediante el desarrollo de una industria competitiva tanto en el interior como fuera del país, para lo cual estableció la creación de infraestructura para esta actividad. Otro de los objetivos del programa está en relación con el fomento al turismo de las regiones fronterizas con el fin de aumentar las divisas provenientes del mismo y atenuar el desequilibrio comercial externo.

\footnotetext{
5 Ladman, Jerry R. op. cit, p, 130

${ }^{6}$ Véase: Raúl A. Fernández. La Frontera México-Estados tinidos. Un estudio socioeconómico. Ed. Terra Nova, Mexico, 1980. p. 132
} 
"Aunque el PRONAF tuvo un éxito inicial, pronto mostró que no era suficiente para satisfacer la situación de desempleo en el área, por lo que en 1965 el gobierno mexicano estableció el Programa Industrial Fronterizo (PIF) que entró en funciones en 1967" 7, el cual básicamente consiste en el establecimiento de plantas maquiladoras para alcanzar los objetivos de;

a) Fomentar la creación de empleos.

b) Mejorar el nivel de vida de la población.

c) Introducir métodos modernos de manufacturas.

d) Capacitar mano de obra.

e) Incrementar el consumo de materias primas del país.

f) Reducir el déficit comercial.

Lo atractivo del PIF consiste en las facilidades otorgadas, en materia fiscal, al capital extranjero para realizar parte del proceso de producción dentro del territorio nacional - esta parte de la producción corresponde a la etapa de mayor utilización de fuerza de trabajo-; los estímulos fiscales emanan de dos acuerdos presidenciales que entraron en vigor en abril de 1971, que consisten en liberar a los inversionistas extranjeros del pago de derechos de aduana y reglamentos sobre importación de materias primas, maquinaria y equipo necesarios para el funcionamiento de las maquiladoras; así también, exceptúa a la zona fronteriza de las restricciones establecidas al capital extranjero, siempre que se invierta en esta región y que el $100 \%$ de su producción sea exportada.

Por su parte el gobierno norteamericano colaboró con el PIF estipulando en su código arancelario (artículos 806 y 807) el permiso a las corporaciones estadounidenses para reimportar libres de impuesto, a excepción de aquellos aplicados al valor agregado por la mano de obra extranjera, ciertos artículos ensamblados o armados en el exterior.

Pero a pesar de que el principal objetivo de la implantación de maquiladoras fue el de crear empleo y cubrir un exceso de oferta de trabajo originada por la baja de las actividades agropecuarias y la constante migración, al analizar la estructura ocupacional de las maquiladoras tenemos que más de dos tercios de sus empleados son mujeres, y por consecuencia, no podemos afirmar que da ocupación a la población desplazada del campo -tanto local como nacional- ya que el trabajo agrícola se realiza casi en su totalidad por hombres.

Otro de los argumentos utilizados para el establecimiento del PIF fue el de fomentar el consumo de materias primas nacionales, pero al mismo tiempo se otorgaron facilidades para la importación de materias primas a quienes las procesaran en la zona fronteriza, con la condición de que el total de la producción se exportara, dando como resultado que no existe tal incremento del consumo de materias primas nacionales. Igualmente resulta dudoso que el funcionamiento de las maquiladoras sirva como capacitación

7ldem. p. 146 
y adiestramiento del personal, ya que generalmente estas empresas trabajan con una planta gemela en Estados Unidos, que es la que proporciona el personal técníco altamente capacitado en el manejo de maquinaria y es muy común que éstos sean los encargados del desarrollo y supervisión de la producción así como del mantenimiento de la maquinaria. Además, el adiestramiento del personal obrero es en base al tipo de equipo con el que trabajan, que por lo regular no es el más moderno, por lo que la capacitación se realiza en base a equipo obsoleto.

Por otra parte, la implantación de empresas maquiladoras ha propiciado y facilitado la penetración de capital extranjero fortaleciendo los vínculos de dependencia con los Estados Unidos, en contra de la tradición política de México, al menos teóricamente, encaminada al fortalecimiento nacional, así como la contención a la inversión extranjera.

\section{EL ASENTAMIENTO HUMANO IRREGULAR Y LA REGION FRONTERIZA.}

Actualmente Baja California no es ajena a las consecuencias generadas por la crisis nacional sufrida en la década de los setenta y agudizada en la presente, de la cual fue partícipe en dos sentidos: a) la caída de la producción agrícola del valle de Mexicali, $y$ b) al ser Baja California receptora de un gran porcentaje de la fuerza de trabajo expulsada por el proceso de industrialización nacional y de la pauperización del campesinado, que ha sido el sector a costa del cual se ha efectuado esta industrialización.

Las características en la producción de la región fronteriza en Baja.California se muestran a través de la estrecha vinculación y dependencia con respecto a Estados Unidos; el sector primario se ve influenciado por la creciente utilización de maquinaria de origen extranjero, ya que internamente no se produce, lo cual si bien permite elevar los volúmenes de producción, también implica la importación de tecnologías. Algo similar ocurre en el sector secundario, pero éste se ve no sólo influenciado por la tecnología, sino que la dependencia se manifiesta también en el origen del capital que lo hace funcionar; además, hay que señalar que la población fronteriza tiene muy arraigados hábitos de consumo de productos manufacturados en los Estados Unidos.

Lo que es importante destacar es que la región fronteriza del Norte en general es reflejo del tipo de relaciones entre Mexico y Estados Unidos; este ultimo, país capitalista del cual el primero es dependiente, y que los problemas en la frontera, al igual que los del resto del país, no pueden ser resueltos a nivel local o en sólo un aspecto, sino a través de cambios estructurales que establezcan otro tipo de relaciones entre ambos países; pero lo más importante es que estén encaminados a solucionar los problemas que generan la desigual distribución de la riqueza que actualmente caracteriza a México y que da origen a la agudización de las contradicciones entre las clases que, de alguna forma, tarde o temprano tienen que ser resueltas. 
En este marco, la migración, así como la conformación de asentamientos humanos irregulares, son fenómenos sociales generados por las necesidades propias del proceso de acumulación capitalista fundado en el cambio cualitativo y cuantitativo de composición orgánica de capital, a través de un repetido incremento de capital constante (medios de producción) a expensas del capital variable, generando un excedente de población (oferta de fuerza de trabajo) llamado ejército industrial de reserva, que se caracteriza por la inseguridad en cuanto a empleo, vivienda, alimentación, salud, educación, etc.

La existencia de este excedente de población asegura al capital su fortalecimiento y continuidad; siendo al mismo tiempo inherente al modo de producción capitalista, su importancia se deriva de la disposición de éste al capital ya sea en momentos de auge o,por el contrario,en crisis.

En este contexto, el proceso migratorio y el surgimiento de asentamientos humanos irregulares, representan el grado de expansión de la oferta de fuerza de trabajo (ejército industrial de reserva) en el mercado capitalista, contribuyendo con ésto a mantener más bajos los salarios y facilitando un crecimiento económico basado en fuertes desigualdades sociales y altos grados de concentración de capital.

\section{CARACTERISTICAS DEL ASENTAMIENTO HUMANO IRREGULAR (A.H.I.).}

Las características de los asentamientos en estudio, a los cuales se les ha denominado de diversas formas tales como asentamientos espontáneos, ciudades perdidas, cinturones de miseria y zonas marginadas, presentan en suma una serie de particularidades que expresan en general un mismo fenómeno social; cualesquiera que sea la denominación que se les otorgue, se caracterizan fundamentalmente por ser zonas habitacionales en donde predomina la población de origen rural, originados por ocupación o invasión organizada de terrenos cuyo carácter en la tenencia del suelo es ambiguo ya que debido al bajo poder adquisitivo de la población que reside en estos asentamientos, sólo pueden adquirir el suelo mediante los siguientes mecanismos " $\ldots .1$. Invasión, 2. Arreglo o compra con ejidatarios... y o comuneros. .,; 3. Ocupación pacífica y permitida por varios lustros...; 4. Compra o alquiler de terrenos sin servicios y apenas si lotificados, pero muy baratos (algunas veces el que les vendió lo está haciendo ilegalmente); . . 5. Predios detentados como parte del salario y a manera de prestación social (campamentos de los. constructoes). .."8, con ausencia de diseño urbano tradicional aunado al predominio del uso de materiales flamables y de desecho para la construcción de vivienda, así como carencia de infraestructura y equipamiento.

La población que reside en estos asentamientos paradójicamente se establece en zonas no urbanizadas, en las que al correr del tiempo y con el

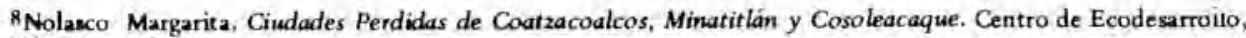
México. p. 55 . 
esfuerzo de sus habitantes por la introducción de servicios mínimos, van creando un espacio urbanizado, resultando una mercancía valiosa con urbanización precaria. "... Esta mercancía más o menos valiosa no puede ser conservada por ellos siempre en situación precaria, por lo que la venden a otros más privilegiados que ellos (es decir, con menos posibilidades de empleo precario), y se pasan a otro terreno pero situado (más contanninado, más peligrosamente cercano a los complejos industriales), sin servicios, sin habitacion y ahí repiten el proceso..." 9 de valorización del suelo.

Al interior de estos asentamientos con características sui generis que los diferencían del resto de los asentamientos establecidos en las zonas urbanas, reside una población la cual ha sido objeto de diversas designaciones tales como pobres de la ciudad, precarios, proletarios, marginados, y miserables; indistintamente, esta población presenta las siguientes características: a) son habitantes de zonas urbanas y "... metropolitanas y lo han sido durante períodos variables de tiempo. . . b) no tienen una posición económica clara o definida en el sistema urbano de clases; c) habitan en asentamientos. .. formados en base a la ocupación ilegal de terrenos públicos o privados. .." 10 .

\section{CONDICIONES ESPECIFICAS.}

Particularmente, las características propias de los AHI de la ciudad de Mexicali no demuestran diferencias significativas con respecto a los asentamientos mencionados anteriormente; sin embargo, debido a su ubicación fronteriza es pertinente mencionar algunos rasgos específicos de éstos: el carácter ambiguo de la tenencia de suelo es originado básicamente por ocupación o invasión de terrenos ubicados en propiedad ejidal, gubernamental y privada.

En el momento en que el suelo adquiere valor, es decir, cuando es mínimamente urbanizado, el Estado interviene con mecanismos a los que el posesionario no puede dar respuesta económica para continuar en este, siendo así desalojado de la zona, introduciendo el Estado de esta manera un aumento de urbanización para después dar cabida a aquellos que puedan solventar su ocupación.

Los materiales de construcción de la vivienda son predominantemente de adobe y materiales flamables o de desecho (cartón, madera, llantas y láminas), este tipo de construcción se debe fundamentalmente al estado jurídico del suelo, que representa un peligro constante de desalojo del terreno $y$, por ende, de su vivienda.

La ausencia de servicios (agua, drenaje, electricidad, servicios médicos asistenciales, etc.) son características de los AHI.

\footnotetext{
${ }^{9}$ Idem. p. 58

10 Montaño, Jorge. Los Pobres de la Ciudad de los Asentamientos Espontáneos. p. 41.
} 
Finalmente, la población migrante que radica en los AHI es predominantemente de origen rural; una parte de esta población se caracteriza por ser ejército industrial de reserva, implicando con ello limitantes para la incorporación de la fuerza de trabajo en la estructura económica, hecho que redunda en precarias condiciones de vida.

\section{MIGRACION Y FUERZA DE TRABAJO EN LOS ASENTAMIENTOS HUMANOS IRREGULARES.}

El papel que han jugado las migraciones internas en México ha representado un movimiento de población de tal magnitud que en la década del 60-70 alrededor del $8 \%$ de la población del país se encontró involucrada en la migración interregional. Se trata de una migración que en su mayoría se caracteriza por ser de tipo rural-urbana. "... En contraste con la migración internacional de la población del país hacia los Estados Unidos se observó que en la década mencionada anteriormente, ésta no alcanzó ni el $10 \%$ con respecto a las migraciones internas de tal forma que esta última, representó aproximadamente, el $90 \%$ de los movimientos migratorios. . ."11

La capitalización de la agricultura, el desarrollo industrial, la problemática en la adquisición de suelo y vivienda, la diferenciación marcada entre campo y ciudad, la situación salarial México-Estados Unidos, y el desarrollo urbano de la franja fronteriza en ambos lados de la frontera, son elementos todos que actúan como factores de migración. En este contexto, la migración ha desempeñado un papel importante en la conformación de los asentamientos humanos fronterizos.

". . la urbanización del norte de México y la urbanización hacia el norte son inseparables. Mientras que la migración de todo tipo determinó en gran parte los asentamientos urbanos del área fronteriza, actualmente dicha área se ha transformado en fuente de una nueva $\mathrm{e}$ importante forma de migración..."12.

A partir de la investigación de campo desarrollada en el proyecto de investigación "Asentamientos Humanos Irregulares en Baja California", se identificó la información que a través de su análisis permitiría conocer algunas de las características del flujo migratorio hacia los asentamientos humanos irregulares de la zona considerando los factores de rechazo y atracción migratoria, el número de miembros de familia que realiza la migración, identificando al mismo tiempo las residencias de los migrantes en lugares intermedios y año de llegada a la ciudad de Mexicali.

Parte de la población que reside en este tipo de asentamiento es migrante, la cual por diversas causas (factores de cambio o de estancamiento)

11 Secretaría de Programación y Presupuesto. Reseña Comentada sobre Estudios de Migración en México, S.P.P. Noviembre de 1980. pp. 29-30.

12 Fernández, Raúl A. op. cit. p. 117. 
decidió trasladarse hacia Baja California con perspectivas de obtener mejores condiciones de vida. En general, los factores de expulsión que presentaron mayor concentración de población migrante de estos asentamientos son: a) migra la familia, en el $32.5 \%$ de los casos (1,185 migrantes); 2 ) incapacidad de explotar ciertos recursos, representando un $22.3 \%(813)$; y 3 ) falta de tierra y/o pobreza de la misma,con un $11.3 \%$ (411). Cabe mencionar que el $18.9 \%$ o (691) de los jefes de familia migrantes corresponde a otros factores de expulsión.

En relación a los factores de atracción, 1,067 migrantes expresaron que su principal motivo para migrar hacia el estado de Baja California fue debido a que en éste contaban con la estancia de familiares o amigos, en un $32.6 \%$ $(1,067)$; el $25.3 \%(828)$ de esta población se trasladó en busca de mejores condiciones de vida; el 11.8 \% (387) tenía intenciones de emigrar a Estados Unidos; y el $10.5 \%$ (342) contaba con un ofrecimiento de trabajo previamente asegurado; el 19,8\% (648 migrantes) manifestaron otros motivos para migrar.

Otra de las características en el proceso migratorio hacia Baja California es la movilización que se desarrolla y que bien puede ser de forma individual o colectiva; nos referimos aquí específicamente a que si el migrante se traslada en compañía de otras personas o solo. De los 3,646 migrantes en estudio, $67.0 \%(2,444)$ afirmaron haber migrado hacia el estado en compañía del jefe de familia, esposa, hijo (s), otros parientes o con amigos; por otra parte, el porcentaje de migrantes que expresaron haberlo hecho en forma individual asciende al $21.4 \%$ (778).

Con relación a la residencia en lugares intermedios con frecuencia única o múltiple, se determinó a partir del tiempo de exposición del migrante, por más de tres meses en la residencia intermedia antes de llegar a su lugar de destino, en este caso hacia Baja California, representando un $29 \%(1,058)$. Los lugares que se caracterizaron por captar población migrante en residencias intermedias, ya sea única o múltiple, fueron: Sonora, Estados Unidos, Baja California, Sinaloa, Jalisco y el Distrito Federal. Asimismo, la migración hacia Baja California en su mayor parte fue directa $71 \%(2,588 \mathrm{mi}$ grantes); esto significa que el estado representa una atracción migratoria debido a que fundamentalmente se caracteriza por ser una región fronteri$\mathrm{za}$, que juega un doble papel en el sentido de que, por una parte, esta situación sugiere una posibilidad de cruzar la frontera México-Estados Unidos; y por otra,merced a la dinámica propia del desarrollo urbano del estado, generado por las políticas gubernamentales y la inversión extranjera en cuanto a desarrollo industrial se refiere.

El análisis del fenómeno migratorio en la ciudad de Mexicali se vierte particularmente en los asentamientos irregulares, los cuales fueron formados fundamentalmente por población migrante. Dichos asentamientos aparecieron por vez primera en el año de 1934, con la fundación del poblado Compuertas. A partir de esta fecha y hasta 1960 se consolidaron 13 asentamien- 
tos irregulares; cabe mencionar que en este período el $56.8 \%$ de los actuales jefes de familia $(1,833)$ participaron en esta conformación.

A partir de 1961 y hasta 1982 , aparecieron 13 asentamientos más, creados también en su mayor parte por población migrante, ya que durante este período se establecieron 1,393 jefes de familia migrantes (43.2\%).

El mayor incremento de flujo migratorio en los AHI se registró en el primer período ya mencionado, coincidiendo éste con la aparición de los Convenios de Braceros establecidos en el año de 1942 y finalizados en 1964, cuyo objetivo consistía en captar mano de obra mexicana requerida por los Estados Unidos, principalmente para el desarrollo de la producción agrícola. Otro elemento que da respuesta a este flujo migratorio consiste en el desarrollo agrícola del valle de Mexicali con el cultivo del algodón.

Actulmente los asentamientos humanos irregulares se caracterizan, por una parte, por la posesión ilegal de la tenencia del suelo, producto de la incapacidad adquisitiva de esta parte de la población para establecer la relación de compra-venta en el mercado de suelo; y por otra, por la necesidad esencial de un habitat,presente en todo ser humano.

Desde su fundación hasta la actualidad se han formado 26 asentamientos con carácter de irregular, en donde habita una población de 21679 habitantes, constituida por 3,707 familias (en el $98 \%$ de éstas el jefe de familia es migrante) distribuidas en 3, 646 viviendas.

La distribución de la estructura poblacional de los AHI asciende a 10,833 hombres y 10,796 mujeres, de los cuales 13,444 son nativos $(62.1 \%)$ y 8,235 son migrantes $(37.9)$.

Cabe aclarar que en la pirámide poblacional, específicamente en el rango de edad de 0-14 años, se encuentra una población total de 8,954 (41.3\%) de los cuales $7,846(36.1 \%)$ son nativos y $1,108(5.0 \%)$ son migrantes. Es importante resaltar este dato ya que en este rango de edad se concentra la mayor parte de la población nativa en tanto que, por el contrario, para la poblacion migrante su mayor concentración se encuentra en la parte superior de la pirámide (representada por los jefes de familia) constituyendo un total de 3,646 jefes de familia, y de donde podemos inferir que el porcentaje anteriormente mencionado para nativos comprendid $\phi s$ en el rango de edad 0-14 años, es en su mayoría, de migrantes.

\section{ANALISIS SOCIO-OCUPACIONAL ENTRE POBLACION MIGRANTE Y NATIVA.}

Con el objeto de identificar las diferencias o similitudes que se presentan entre nativos y migrantes, se hace necesario un análisis socio-ocupacional, que nos permitirá en primera instancia identificar el grado de incorpo- 
ración de esta población al mercado laboral urbano, así como conocer el nivel de competividad que existiera entre sí.

La población ocupada en los asentamientos humanos irregulares asciende a 5,736 personas, de las cuales $3,571(62,2 \%)$ son migrantes y 2,165 $(37.8 \%)$ son nativos.

La participación de los migrantes en la estructura económica asciende en el sector primario a $541(9.4 \%)$ ocupados, en el secundario a 1,258 $(21.9 \%)$, y en el terciario $1,772(30.9 \%)$. Con respecto a los nativos, éstos se caracterizaron por la no incorporación al sector primario, concentrándose en el sector secundario con $909(15.8 \%)$ y en el terciario agrupando a $1,256(22.0 \%)$.

La incorporación de migrantes y nativos en la estructura económica no demuestra diferencias significativas a excepción de la rama de actividad agrícola, en la que los migrantes se incorporan a 9.0 por ciento (519), situación que se explica en gran parte debido al origen rural de la población que está directamente relacionada con la producción agrícola del valle de Mexicali. Por el contrario, la población nativa no presentó la incorporación a esta rama de actividad debido en parte a la dinamica urbana de la economía fronteriza.

En relación a las demás actividades, se manifiestan similitudes en la incorporación a la estructura económica entre nativos y migrantes, presentándose una concentración en las actividades de servicios, construccion, comercio y transporte, predominando entre éstas la actividad de servicios. Esto se explica en la medida en que es el desarrollo industrial el que genera actividades complementarias, incrementándose con ésto el crecimiento del sector terciario, necesario para el propio desarrollo industrial.

Por otra parte, el desarrollo industrial genera al mismo tiempo no sólo el crecimiento del sector terciario, sino también el crecimiento de un sector poblacional excedentario (es decir el ejército industrial de reserva) y el incremento en los flujos migratorios.

Ya Marx en su Crítica a la Economía Política afirmaba que el capitalismo requiere no sólo del crecimiento natural, sino también del crecimiento social de la población. Y al mismo tiempo tiene la necesidad de producir una población excedentaria respecto de la necesidad media de valorización del capital, que es una condición vital de la industria contemporánea 13 .

\section{MIGRACION Y ANALISIS SOCIO-OCUPACIONAL}

Para desarrollar este apartado se hizo un seguimiento de los antecedentes laborales del migrante, identificando para ello el lugar de origen y la ocu-

${ }^{13}$ Véane: Carlos Marx. El Capital, Tomo 1, Vol. 3 pp. 788-789. 
pación principal que desarrollaba en el mismo, así como el primer trabajo que efectuó en la ciudad de destino, en este caso la ciudad de Mexicali.

Los resultados obtenidos se procesaron de tal forma que permitieron identificar las 6 entidades federativas, de las 31 existentes en el país, que presentaron mayor expulsión de población.

Como primera instancia, dicho seguimiento laboral partió del análisis socio-ocupacional de cada una de las actividades desarrolladas por el migrante en su lugar de origen, obteniendo las cinco actividades más representativas en las que se concentró la mayor parte de esta población $(94.1 \%)$.

El volumen de población total ocupada asciende a $2,180(100 \%)$ cubriendo a las 31 entidades federativas que se especifican en el estudio. Las actividades a que nos referimos en el porcentaje anterior son las siguientes: 1) agricultura $1,436(65.9 \%) ; 2)$ servicios $217(9.9 \%)$ transformación 181 $(8.4 \%) ; 4)$ comercio $110(5.0 \%)$ y 5$)$ construcción $108(4.9 \%)$.

Lo anterior nos permite hacer las siguientes apreciaciones: primero, la actividad laboral que presentó mayor concentración de población fue la agricultura con $1,436(65.9 \%)$. Consideramos que una de las razones para que la población que trabajaba en este sector migrara hacia Baja California se debio, en términos generales, a que los salarios mínimos establecidos para las zonas rurales eran inferiores a los salarios mínimos establecidos para las zonas urbanas.

De lo anterior se desprende que efectivamente, bajo estas circunstancias de desigualdad social, se manifiesta, como lo han afirmado la generalidad de los estudios sobre migraciones internas, que es la migración rural-urbana la que se presenta con mayor frecuencia en relación a los demás tipos de migraciones señaladas, aunque no por ello deja de ser importante el estudio de las demás modalidades de migración. En ese sentido, es necesario explicar las circunstancias que hacen posible la disminución de los salarios mínimos para las zonas rurales con respecto a las urbanas.

"El costo de producción y de reproducción de la fuerza de trabajo es menor en el sector rural que el urbano. Ello se debe a la frugalidad de los insumos en la economía campesina, que expresan la baja retribución que en el interior de esa economía recibe el trabajo insumido" 14

Es necesario señalar que, en general, el valor de la fuerza de trabajo está directamente determinado por el tiempo de trabajo socialmente necesario para su producción y reproducción. Sin embargo, "El campesino se encuentra en una situación especial. En tanto que produce la mayor parte de los bienes que consume, el valor de la fuerza de trabajo que produce y vende emana

14 Margulis, Mario. Contradicciones en la Estructura agraria y transferencias de valor, El Colegio de México. p. 92. 
en forma directa de la productividad en su propia rama de actividad. La baja productividad en el interior del sector campesino - producto de la baja composición orgánica del capital - se contrapone y complementa con la frugalidad de la canasta de bienes que consume y con el hecho de que puede obtener la mayor parte de sus insumos sin tener que adquirirlos en el mercado, con lo cual evita las ganancias normales y las especulativas incluidas en los precios (. . . ) De modo que no es que el campesino produzca su fuerza de trabajo a un costo menor, sino que ese "costo", medido en tiempo de trabajo, es muy elevado. La baja productividad en este sector exige muchas horas de trabajo para producir las mismas subsistencias (...) Así igualada o corregido de trabajo, encontramos una ventaja relativa en la mayor frugalidad de los consumos del sector campesino, que permite producir fuerza de trabajo con una menor canasta de bienes y también en relativa independencia del mercado al producir buena parte de los bienes que consume, con lo cual se evitan pérdidas de valor en el plano de la circulación (...) De modo que el costo de producción y parte del costo de reproducción de la fuerza de trabajo campesina son transferidos en forma gratuita al sector capitalista" 15

En la medida en que la mayor parte del consumo de la familia del asalariado agrícola sea costeada por la unidad familiar, en esa proporción el salario de los trabajadores agrícolas irá en decremento.

"En resumen: en la intimidad del bajo salario pagado a los trabajadores provenientes del sector campesino, se oculta uno de los principales mecanismos de transferencia de valor desde ese sector al capitalista. El sector capitalista, en especial el agrario, se beneficia con los bajos precios de la fuerza de trabajo ya que una parte del valor de ésta es costeada por el propio sector campesino (mantenimiento de la familia), costo de producción de la fuerza de trabajo hasta llegar a la edad activa, mantenimiento en épocas del año en que los ritmos agrícolas requieren menos trabajo asalariado).

"Este proceso, que reduce el precio de la fuerza de trabajo y aumenta la plusvalía en el sector capitalista, depende de la esfera de la circulación, pero lo que se compra es resultado de un proceso de producción. Y es este proceso de producción, que llamamos de subsistencia, el que hace posible - por sus características, en especial por la baja remuneración del esfuerzo laboral que se desarrolla en su interior- esta transferencia a título gratuito de valor hacia el sector capitalista" 16

Segundo: las ocupaciones en las ramas de actividad de transformación, servicios, comercio y construcción, que ascienden a 28.2 por ciento $\left(616 \mathrm{mi}^{-}\right.$ grantes), demuestra que en esta parte de la población, por sus características, su incorporación al mercado laboral bien puede ser en la manufactura o con frecuencia en el sector terciario, en tanto que en este último la exigencia de

\footnotetext{
${ }^{15}$ Idem. pp. 94-96.
}

16Idem. Pp. 96-97 
especialización laboral para ejercer no es determinante en relación a las ocupaciones directamente vinculadas con la industria.

"El rápido crecimiento del sector terciario se debe a que las ciudades, sede de la expansión industrial, tienen que proveer al sector industrial una infraestructura de servicios con la que no contaban. Además, como el crecimiento industrial se inicia con una base de acumulación relativamente pobre no permite que los servicios se desarrollen con una capitalización intensa simultánea, de tal forma que compitan con la industria por los escasos fondos disponibles. Por lo tanto, los servicios se expanden favorecidos por la abundante mano de obra y no constituyen un obstáculo para el crecimiento capitalista" 17

Tercero: las entidades federativas que absorbieron mayor población en todas las actividades productivas se caracterizaron al mismo tiempo por ser los estados de mayor expulsión de población migrante hacia Baja California; nos referimos particularmente a Jalisco, Sinaloa, Sonora, Michoacán, Guanajuato y Zacatecas.

Por otra parte, es importante señalar la primera ocupación del migrante en la ciudad de Mexicali, con el objeto de enmarcar la experiencia ocupacional del migrante en relación a la incorporación de éste al mercado laboral, estableciendo criterios comparativos con la ocupación en su lugar de origen y en el lugar de destino del mismo.

Los 3,646 actuales jefes de familia migrantes, al incorporarse al mercado laboral en la ciudad, realizaron las ocupaciones de servicios donde laboraron 803 migrantes $(22.07 \%$ ); en agricultura $475(13.0 \%)$; la ocupación de comercio absorbió $389(10.77 \%$ o) ; construcción $382(10.5 \%$ o) y transformación con $347(9.57 \%$ o). Dichas ocupaciones, al ser las más representativas en cuanto a absorción de fuerza de trabajo, permitieron hacer algunas aseveraciones, estableciendo las comparaciones ocupacionales entre la realizada en su lugar de origen y la primer ocupación en su lugar de destino: 1) coinciden con ser las actividades predominantes de absorción en ambas regiones; 2) la ocupación de agricultura captó mayor población en su lugar de origen $(65.9 \%) ; 3)$ servicios fue la ocupación que absorbió más población migrante en su lugar de destino (ciudad de Mexicali) con un $22.0 \%$ o; 4 ) comercio, ocupación que en su lugar de origen fue de un $5.0 \%$, en la ciudad de Mexicali tuvo un incremento mayor ascendiendo a un $10.7 \%$ y 5) la ocupación de transformación mostro mínimas diferencias en las dos zonas mencionadas.

\footnotetext{
17 Humberto Muñoz y Orlandina Oliveira. "Algunas Controversias sobre la Fuerza de Trabajo en América Latina". En: Fuerza de Trabajo y Movimientos Laborales en América Latina. p. 29.
} 


\section{INCORPORACION DE LA FUERZA DE TRABAJO A' LA POBLACION ECONOMICAMENTE ACTIVA EN LOS ASENTAMIENTOS HUMANOS IRREGUL.ARES.}

La población económicamente activa (PEA), es aquella parte de la población que proporciona tanto el capital como la mano de obra para la producción de bienes y servicios de índole económico, incluyendo a los empleadores, a las personas que trabajan por cuenta propia, a los trabajadores familiares no remunerados, a los asalariados, y a los desocupados que declaran tener un oficio o profesión 18

La PEA es una parte de la población total de un país, que se distingue fundamentalmente por las siguientes características:

1). Porque potencialmente puede desempeñar un trabajo, el cual se determina en función de la edad.

2) Por la capacidad física y mental para trabajar.

3) Por la disponibilidad de tiempo para hacerlo.

La PEA se manifiesta al interior de la estructura socioeconómica bajo dos modalidades: a) población ocupada, y b) población desocupada 19 La primera aglutina a la población que cuenta con un empleo específico; la segunda, agrupa a todos los desocupados, los cuales a su vez se subdividen en desocupados abiertos y encubiertos. Los desocubados abiertos son aquellos que efectúan trámites para conseguir trabajo (como consultar agencias de empleo, sindicatos, empresas, amistades y familiares); los desocupados encubiertos son aquellos que no realizan ningún trámite para obtener algún empleo, pero están dispuestos a aceptarlo en caso de que se les propusiera.

Cabe mencionar que los rangos de edad establecidos en los censos en relación a la PEA han variado en el tiempo. Por ejemplo, en los censos de 1950 la PEA se consideró a partir del rango de edad de los 8 o más años; en el censo de 1960 y 1970 , de los 12 años o más, rango que se mantuvo en la encuesta continua sobre ocupación en 197820 y actualmente las estadísticas laborales de México establecen que la edad suficiente necesaria para trabajar es a partir de los 12 años y más (X Censo General de Población y Vivienda).

\footnotetext{
18 Véase: Secretaría de Programación y Presupuesto, La Población de México, su ocupación y sus nivelés de bienestar. Serie: Manuales de Información Básica de la Nación. Coordinación General del Sistema Nacional de Información. México 1980. p. 69.

19 Idem: p, 61 .

20 Idem. p. 69.
} 
Específicamente en el estudio se consideró sólo una parte de la PEA, que se refiere a la oferta de fuerza de trabajo, partiendo de las características mencionadas en cuanto a edad suficiente y capacidad física y mental para trabajar, ubicando a la PEA en el rango de 15 a 64 años de edad 21.

Para identificar a la PEA (retomando de ésta la parte correspondiente a la oferta y demanda de fuerza de trabajo), partimos del análisis de la población ocupada y desocupada existente en los AHI.

La población total de estos asentamientos asciende a 21,679 habitantes, de los cuales la población ocupada es de $5,376(26.5 \%$ o $)$ y la población desocupada de $7,037(32.5 \%)$, correspondiendo $8,906(41.0 \%$ o) a la población infantil de 0-14 años. De la población ocupada, 4,552 $(79.3 \%$ o) son hombres y $1,184(20.7 \%$ ) son mujeres. Con respecto a la población desocupada se identificó un porcentaje inferior de hombres $(25.7 \%$ o sea 1,806$)$ con relación a las mujeres $(74.3 \%$ o sea 5,231$)$.

Sin embargo, la edad necesaria para la incorporación de la fuerza de trabajo a la PEA se considera en el rango de edad de los 15-64 años. A partir de este criterio, la PEA en los AHI reúne un total de 12,096 habitantes que representan el $55.8 \%$; dicha población está en edad de incorporarse al mercado laboral.

Asimismo, la concentración mayor de la PEA se encuentra en los rangos de 15-19, 20-24, 25-29 y 30-34 años de edad, que reúnen un total de 7,653 habitantes $(63.3 \%$ o); el resto de la población que tiene capacidad potencial para participar en la oferta y demanda de fuerza de trabajo asciende a un $36.7 \%$.

Es necesario hacer notar que la distribución de la PEA ocupada y desocupada constituye un volumen dê 5,590 habitantes $(46.2 \%$ ) y 6,506 $(53.8 \%)$ respectivamente.

Los datos anteriores permiten hacer la siguiente reflexión: el porcentaje de los desocupados en los AHI, es superior al de los ocupados; esta información hace afirmativa una de las hipótesis que se desarrolló en el estudio, en el sentido de argumentar que son los habitantes de estos asentamientos los que participan directamente para la formación y reproducción del ejército industrial de reserva que garantiza al mismo tiempo la acumulación de capital.

La incorporación de la población ocupada al mercado de trabajo presenta dos formas que predominan como mecanismos para su absorción, éstos son: a) el trabajo permanente, y b) el trabajo eventual. El trabajo permanente se caracteriza por la "estabilidad jurídica" que se establece en las relacio-

21 Vlase: Naciones Unidan. Demographic Yearbook 1961. (1962 New York). International Labor Office. Yearbook of Lebor Statica. 1962. 
nes obrero-patronales (condiciones mínimas necesarias para la reproducción de la fuerza de trabajo establecida por la Ley Federal del Trabajo), como son la jornada de 8 horas, salarios mínimos, condiciones salubres de trabajo, asistencia médica, y prestaciones sociales en general.

Por el contrarió, los trabajadores eventuales están regidos por un contrato de trabajo definido, en general presentan circunstancias inestables de empleo, salarios y prestaciones sociales.

Específicamente, en los AHI estas dos formas de trabajo (permanente y eventual) representaron, por una parte, el $49.5 \%$ o $(2,840)$ y por otra, el $50.5 \%$ o $(2,896)$ respectivamente.

En términos generales, la PEA ocupada, ya sea permanente o eventual, se incorpora al mercado laboral con un determinado horario de trabajo cuya vinculación respecto al salario presenta diversas variantes. Al respecto, el número de horas trabajadas diariamente se distribuyó de la siguiente manera: los que trabajan 4 horas o menos son 259 personas $(4.6 \%$ ); los de más de 4 y menos de 8 horas ( 5 a 7 horas) fueron $535(9.6 \%)$; los de ocho horas $3,764(67.3 \%) ; y$ más de ocho horas $1,032(18,5 \%)$.

En cuanto a los salarios que percibe la población ocupada en los AHI, se resumen en los siguientes datos: a) 3,663 personas $(63.8 \%$ o obtienen un ingreso menor al salario mínimo; b) 1,459 (25.4\% recibe el salario mínimo; y c) $614(10.8 \%)$ se agrupan en los niveles de ingreso de dos o más veces el salario mínimo. Lo anterior denota la sobreexplotación de la fuerza de trabajo, ya que el $85.8 \%$ de la población ocupada labora de ocho horas a más, percibiendo un $63.8 \%$ de ellos menos del salario mínimo, a lo cual se suma la inestabilidad en el empleo, lo que demuestra que los salarios son los parámetros de medición que pueden ser utilizados para identificar el grado de absorción de mano de obra y las condiciones reales en las que se encuentran los trabajadores. Al mismo tiempo, el salario mínimo es la expresión de las características que tiene la acupación de la fuerza de trabajo; nos referimos aquí a los trabajadores desempleados y subempleados que hasta hoy han caracterizado a esta parte de los asentamientos humanos.

Es relevante señalar que tanto la Constitución Política de los Estados Unidos Mexicanos, como la Ley Federal del Trabajo, no contemplan legislativamente las formas particulares de incorporación que presentan las características del autoempleo, como es el caso de la poblacion que reside en los $\mathrm{AHI}$, sino que se limita a legislar exclusivamente en materia de relaciones de trabajo obrero-patronales, hecho que permite excluir jurídica y socialmente a estos trabajadores de los derechos sociales que los trabajadores en su conjunto han conquistado.

Así pues, dentro de este marco legal quedan fuera de todo derecho los trabajadores que perciben un ingreso en forma individual en la práctica del autoempleo, formando así la masa de fuerza de trabajo subempleada y 
desempleada, asumiendo por otra parte, la responsabilidad de reproducirse como fuerza de trabajo sin ningún costo social para el capital.

Particularmente, este hecho se relaciona con las condiciones laborales de los trabajadores que obtienen ingresos con la realización de diversas actividades de servicios (vendedores ambulantes, lustrabotas, vigilantes, lavacoches, recolectores de materiales de desecho, etc.), ocupaciones calificadas como autoempleos, las cuales presentan características contradictorias con el marco jurídico antes mencionado.

A continuación se desarrolla la relación que se establece entre el grado de escolaridad y la incorporación de la fuerza de trabajo en los distintos sectores de ocupación.

Con respecto al grado de escolaridad de la población de los AHI, 1,637 personas son analfabetas, o sea un $8.8 \%$ o con relación al total de habitantes. Cabe mencionar que el $10.4 \%$ o $(2,663)$ cuyo rango de edad fluctúa de 0 a 14 años de edad, se encuentra fuera de la actividad escolar.

Es importante caracterizar la relación que se establece entre el proceso de incorporación de la fuerza de trabajo a una determinada ocupación con los grados de escolaridad, ya sea elemental, media o superior, para identificar el papel que esta última ha jugado en la dinámica ocupacional.

En ese sentido, la ocupación en que se concentra la mayor parte de la población es en el sector servicios, 1,622 personas del total de la población con grado de escolaridad $(9.1 \%$ o), y la relación que se establece va desde educación elemental hasta media y superior, correspondiendo la mayor parte de la población que trabaja en este sector al nivel educativo elemental y medio con un total de 1,257 habitantes $(7.0 \%$ o).

En lo referente a la ocupación de transformación, se presenta una situación similar a la ocupación de servicios en relación al nivel educativo, ya que se concentra una población de 1,104 personas $(6.1 \%$ ); particularmente en el nivel elemental y medio son 972 ( $5.4 \% \%$ ).

Para el total de habitantes ocupados en la construcción, predomina el nivel elemental con 629. spersonas $(3.5 \%$ o), y analfabetas con $281(1.6 \%)$.

La actividad de comercio presenta una extensión en todos los niveles educativos (de igual forma que en los servicios y transformación) con 799 habitantes $(4.5 \%)$

Finalmente, los sectores que concentran a la población con un nivel educativo elemental generalizado son: agricultura, extractiva, electricidad y transporte, con un total de 587 personas $(3.3 \%$ ). 
Por otra parte, las actividades de hogar, estudiante, e incapacitado, reúnen un total de 12,155 (el $67.9 \%$ o con respecto al total de la población), y la situación escolar presenta variantes relativas desde elemental hasta superior. Corresponde particularmente para la actividad de estudiante un total de 8,103 personas $(45.3 \%$ en relación al resto de la población ocupada en los empleos y actividades ya mencionadas).

Con base en la descripción anterior, se observa que en el nivel elemental y medio se concentra el mayor volumen de población de los AHI. Esto permite afirmar que la educación no ha sido un factor determinante para que la población se incorpore dentro del mercado laboral a empleos que le redituen un salario específico; sino por el contrario, la educación legitima y al mismo tiempo justifica socialmente las formas de calificación laboral, así como el desempleo y subempleo que se presenta en los AHI.

El grado de escolaridad, la edad, los sectores productivos y las modalidades de incorporación de la fuerza de trabajo al mercado laboral, permiten dar una idea generalizada de las condiciones de vida de la poblacion de los $\mathrm{AHI}$, que se sintetizan en la obtención de un ingreso por debajo del salario mínimo establecido, o en el mejor de los casos, del mínimo.

Finalmente, es importante hacer notar que por lo general, las ciudades especializadas en servicios, tienen como segunda o posterior función predominante a la industria de transformación, como afirma Luis Unikel22, lo cual implica que cuando una ciudad absorbe mucha PEA en las actividades de servicios o comercio, la industria manufacturera tiene mayor importancia relativa, mientras que cuando la ciudad es predominante industrial, también con frecuencia es terciaria, principalmente en las actividades de comercio y transporte, debido que éstas son complementarias a la industria.

También las ciudades que se caracterizan por tener una actividad fundamental en servicios se presentan como las ciudades de mayor atracción de población, en la medida en que estas actividades representan mayor posibilidad de incorporación de la población al mercado laboral en relación a las ciudades cuya actividad económica fundamental es la de industria.

\section{FUERZA DE TRABAJO MASCULINA Y FEMENINA.}

Dentro de la población que conforman los $\mathrm{AHI}$, existe una movilidad de fuerza de trabajo, cuya manifestación se expresa en la PEA; ésta en deterrinados momentos puede ser ocupada o desocupada, ya que se rige en función de la oferta y demanda de trabajo.

Es necesario hacer notar que la PEA está constituida por población masculina y femenina. Actualmente la participación de la mujer ha aumentanotablemente en relación a décadas anteriores debido al desarrollo industrial

ZUnikel. Luis. op. cit. pp. 167-168. 
y a las condiciones económicas deficientes de la familia, que ha generado que algunos miembros de la misma -entre ellos población infantil- ingresen al mercado laboral para lograr la obtención de mayor ingreso que les permita cubrir las necesidades mínimas de bienestar, para finalmente continuar con el proceso de reproducción de la fuerza de trabajo.

Específicamente, esta población (PEA) constituye un total de 12,906 habitantes, de los cuales $5,590(46.2 \%$ o) están ocupados y $6,506(53.8 \%$ ) son desocupados.

En cuanto a su composición por sexo, dentro de la PEA ocupada existen 4,433 hombres $(79.4 \%$ o) y 1,157 mujeres $(20.6 \%$ o); ahora bien; en la PEA desocupada se agrupan 1,650 hombres $(25.4 \%$ o) y, 4,856 mujeres $(74.6 \%)$.

Es importante señalar que tanto la participación de la fuerza de trabajo femenina como la masculina, dentro de las relaciones de producción capitalista, están sujetas a condicionantes propias del desarrollo industrial que se manifiestan en distintas formas de incorporación.

\section{CONCLUSIONES}

1. Las migraciones internas hacia la frontera norte de México están relacionadas directamente con el proceso de industrialización del país. En este contexto, el proceso de urbanización en Baja California se deriva de su situación fronteriza, en donde las ciudades de Baja California se caracterizan por ser instancias de tránsito hacia los Estados Unidos.

2. El crecimiento de las ciudades fronterizas a partir de 1940-1970, fue generado por la expulsión de población de algunas regiones del centro y sur del país, así como también por la atracción que representa esta zona debido a la implementación de políticas agrarias orientadas a la defensa de la pequeña propiedad, otorgamiento de creditos agrarios para el cultivo de la tierra, uso de inversión estatal para el riego en la zona norte y noroeste de México, así como también por darle a la producción agrícola un carácter de mercancía de exportación, que estimularon el proceso de desarrollo urbano de la frontera norte.

3. Las migraciones internas se explican a partir de la organización so cial de la producción, en donde la acumulación de capital y el consecuente desarrollo industrial, tanto en la ciudad como en el campo, generan una población excedentaria denominada ejército industrial de reserva, y cuya característica es ser fuerza de trabajo desempleada y subempleada pero que le es funcional al capital, y que es población residente en los asentamientos humanos irregulares. 
4. La migración rural-urbana permanente se caracterizó por ser el tipo de migración predominante en los asentamientos humanos irregulares de la ciudad de Mexicali, dado que la mayoría de sus habitantes procede de los estados de Jalisco, Guanajuato, Sinaloa, Zacatecas, Michoacan, Durango y Sonora, distinguiéndose por expulsar a gran parte de esta población, así como fuerza de trabajo que estuvo incorporada a la agricultura, resaltando la incapacidad de la estructura agraria para absorberlos.

Es importante señalar que este tipo de migración fue la más sobresaliente a nivel nacional, incrementándose el desplazamiento campo-ciudad en el período $1940-1980$ a 22.4 millones de personas.

5. La migración es un proceso que necesariamente tiene que ser analizado en todas sus expresiones, partiendo de las causas objetivas (situación internacional, nacional y regional), considerando también las causas subjetivas (motivaciones personales del migrante). Podemos así afirmar que socialmente pueden estar dadas condiciones necesarias para migrar, pero si no se manifiestan las condiciones subjetivas o suficientes, es probable que la migración no se desarrolle.

6. Las diversas formas de producción en el agro mexicano (de subsistencia y capitalista), son la expresión del desarrollo desigual del capitalismo que se presenta en el país. En ese sentido, la forma de producción de subsistencia está incorporada y es producto del modo de producción dominante.

7. Uno de los impactos de las migraciones internas en la ciudad de Mexicali es la formación de asentamientos humanos irregulares. Esta afirmación se fundamenta en el sentido de que es propiamente el migrante el generador de dicho asentamiento.

8. La práctica del autoempleo en el asentamiento irregular vislumbra la reproducción de la fuerza de trabajo sin ningún costo social para el capital, en tanto que esta parte de la población percibe ingresos menores al mínimo establecido.

9. E1 ejército industrial de reserva tiene características distorsionadas por su magnitud. Se producen formas de extracción de plusvalía y se multiplican los trabajadores que realizan plusvalía al servicio inmediato del capital comercial, y mediato del capital industrial. Por lo tanto, no existen los llamados marginados. Se genera un proceso de proletarización ampliado y deformado. Todo este se encuentra en diferentes fases según el grado de desarrollo del capitalismo en cada formación social dependiente, pero cuya fase terminal será siempre esta proletarización distorsionada.

10. En el proletariado encontramos productores que no han sido liberados totalmente de sus medios de producción; entre estos proletarios se hallan los "maquileros" y sería un error aducir baja tecnología para calificarlos como incorporados "marginalmente" al proceso de acumulación por 
sus niveles de sobreexplotación. Esto explica que, a pesar de un alto grado de subempleo, la acumulación de capital no se entrampa totalmente.

De esta forma, a pesar de la inmensa masa del ejército industrial de reserva, dicho ejército, a través de innumerables autoempleos, participa de manera muy significativa en la acumulación de capital. Hay que señalar que, de trás del pequeño productor independiente, nos encontramos no un pequeño empresario, sino un trabajador asalariado encubierto. La educación en los niveles populares no es un escalón que posibilite el empleo. La escuela legitima la baja calificación laboral y las formas de desempleo y subempleo.

11. A nivel individual, las familias de los trabajadores no alcanzan a mantenerse con los empleos fijos, eventuales o complementarios. Por eso acuden a las redes sociales de subsistencia, las cuales son aprovechadas por la acumulación de capital puesto que permiten que la mano de obra subsista con bajos salarios, y porque hacen posible cierta ampliación de mercado interno.

Las redes sociales también propician la formación de la infraestructura de las organizaciones populares, pero, por su misma naturaleza, tienen un alcance limitado a los niveles iniciales.

12. La formación de asentamientos humanos irregulares beneficia directamente a la acumulación de capital. Los trabajadores hacinados en la ciudad presionan por suelo urbanizable, generalmente en detrimento de la propiedad campesina. Entran en un espacio de ilegalidad. Esto permite un bajo costo del suelo, con lo que la clase trabajadora, a pesar de su reducido salario, puede conseguir habitación.

13. El capital pretende apoderarse de los mejores espacios habitados por los trabajadores, lo cual redundará en su propio beneficio. La regularización posterior beneficia al capital rentista. La consolidación de los asentamientos humanos irregulares lleva a la postre el aumento del costo de la vivienda, lo cual conduce a un proceso de redensificación que termina necesariamente con la expulsión de los sectores más pauperizados, que se ven en la necesidad de reiniciar este ciclo.

14. El Estado, según las etapas de acumulación, ha tenido capacidad para dar salida a las presiones de los grupos de trabajadores necesitados de vivienda; no obstante, esta capacidad se ha visto obstaculizada por la crisis capitalista. En momentos de menor tensión, los líderes locales soportan el peso político; en los momentos de agudización de la demanda, estos líderes generalmente han dejado de ser funcionales. Sin embargo, el Estado ha seguido contando con las distintas instancias del aparato burocrático que, en juego alternativo y aún en contradicciones menores, ha podido controlar las demandas. 


\section{BIBLIOGRAFIA}

APPENDINI, Kirsten A, y Vania Almeida Salles. Agricultura Capitalista y Agricultura Campesina en México. (Diferencias Regionales en Base al Análisis de Datos Censales). Cuadernos del CES, No. 10, Centro de Estudios Sociológicos, El Colegio de México. México, 1977.

ARIZPE, Lourdes. Migración, Etnicismo y Cambio Económico. (Un estudio sobre migrantes campesinos a la ciudad de México). El Colegio de México. México. 1978).

BALAN, Jorge. "Estructuras Agrarias y Migración Interna en una Perspectiva Histónica: Estudios de Casos Latinoamericanos". En: Revista Mexicana de Sociologia. Vol. XLIII. Enero-Marzo de 1981. UNAM. México, 1981.

BARTRA, Roger. Breve Diccionario de Socioiogia Marxista. Ed. Grijalbo. Colección 70. No. 127. México. 1973.

BARTRA, Roger. Estructuras Agrarias y Clases Sociales en México. Ed. Era, México, 1980. 52 . ed.

BUSTAMANTE, Jorge A. "Pasado y Futuro de la Migración Internacional de los Trabajadores". En: Las Re laciones entre México y Estados Unidos. Ed. Nueva Imagen, México 1980.

DIESBACH, Nicole. El Proceso de Producción Agricola en el Valle de Mexicali. Tesis de licenciatura. E.C. S. y P. de la UABC México. 1977.

FEDER, Ernest. Diario Uno Más Uno. Miércoles 18 de agosto de 1982.

FERNANDEZ, Raúl. La Frontera México-tstados Unidos. Un Estudio Socioeconómico. Ed. Terra Nova, México, 1980.

FUENTES Romero, David F. Asentamientos Humanos Irregulares: Metodología de Análisis. Tesis profesional de licenciatura. E.C.S. y P. de la UABC. México. 1980.

GARCIA BRIGIDA; Humberto Muñoz y Orlandina de Oliveira. Migración, Familia y Fuerza de Trabajo en la ciudad de México. Cuadernos del CES No. 26, Centro de Estudios Sociológicos, El Cologio de México. México 1979.

GOMEZ QUINONEZ, Juan y A. Ríos Bustamante. "La Comunidad Mexicana". En: La Otra Cara de México. el Pueblo Chicano. Ed. El Caballito, México. 1980.

JUAREZ, Antonio. Las Corporaciones Transnacionales y los Trabajadores Mexicanos. Ed. Siglo. XXI, México. 1979.

LADMAN, Jerry R. El Desarrollo de la Economia Regional de Mexicali. Un ejemplo de Crecimiento Impulsado por la Exportación. Universidad Estatal de Arizona. Tempe, Arizona, E.U.A. 1975.

LENIN, V.I. El Desarrollo del Capitalismo en Rusia. Ediciones de Cultura Popular, México, 1977.

LOMNITZ, Larissa A. de. Cómo Sobreviven los Marginados. Ed. Siglo XXI, 1978.

MARGULIS, Mario. Contradicciones en la Estructura Agraria y Transferencia de Valor. El Colegio de México, México, 1979.

MARX, Karl. El Capital. T.1. Ed, F.C.E. México, 1979. 2a. ed.

MARX, Karl. El Capital. T.1., Vol. 1, Ed. Siglo XXI, México, 1979. 92. ed.

MARX, Karl. El Capital. Libro I, Capítulo VI (inédito). Ed. Siglo XXI, México, 1980, 8a, ed.

MrTCHELL, Juliet. "Familia, Producción, Reproducción, Sexualidad y Socialización de los Niños". Revista FEM, Vol. 11, No. 7 México, 1976.

MONTIEL, Miguel. "Un Perfil del Pueblo Chicano". En: La Otra Cara de México: It Pueblo Chicano. Ed. El Caballito, México. 1980.

MONTAÑO, Jorge. Los Pobres de la Ciudad en los Asentamientos Espontáneos. Ed. Siglo XXI, México. 1979. $2 d$. ed.

MUNOOZ, Humberto y Orlandina de Oliveira. "Algunas Controversias Sobre la Fuerza de Trabajo en América Latina". En: Fuerza de Trabajo y Morimientos Laborales en América Latina. El Colegio de México, México, 1979.

NACINES UNIDAS. Demographic Yearbook 1961. (1962 New York). International Labor Office Yearbook of Labor. Statistic, 1962. Ginebra, 1962.

NOLASCO, Margarita. Ciudades Perdidas de Coatzacoalcos, Minatitlan y Cosoleacaque. Centro de Ecodesarrollo, México, 1979.

OLIVEIRA, Orlandina de. Migración y Absorción de Mano de Obra en la Ciudad de México: 1930-1970. Cuadernos del CES, Centro de Estudios Sociológicos, El Colegio de México, 1977, 2a. ed.

RESTREPO, Iván. Conflicto entre Ciudad y Campo en América Latina. Ed. Nueva Imagen, México, 1980.

ROJAS Soriano, Raúl. Guia para realizar investigaciones sociales. U.N.A.M., México, 1979.4a. ed.

SECRETARIA DE PROGRAMACION Y PRESUPUESTO. Reseña Comentada sobre Estudios de Migración en México. Sría. de Programación y Presupuesto, México. Noviembre de 1980.

SECRETARIA DE PROGRAMACION Y PRESUPUESTO. La Población de México, su Ocupación y sus Niveles de Bienestar. Serie: Manuales de Información Básica de la Nación. S.P.P. Coordinación General del Sistema Nacional de Información, México, 1980.

ST A VENHAGEN, Rodolfo, Las Clases Sociales en las Sociedades Agrarias. Ed. Siglo XXI, 1980, 12a. ed.

ST AERN, Claudio. Las Migraciones Rural-Urbanas. Cuadernos del CES No. 2, Centro de Estudios Sociológicos. El Colegio de México, México, 1976. 2da. ed.

-NIK EL. Luis. El Desarrollo Urbano de México. El Colegio de México, México, 1978 2da. ed.

¿RZUA, Raúl. El Desarrollo y la Población en A mérica Latina. Ed. Siglo XXI-PISPAL México, 1979. 